\title{
Combinatorial Search for Plasmonic and Epsilon-Near-Zero Chalcogenide Alloys
}

\author{
D. Piccinotti1, B. Gholipour'1, 2, J. Yao², K. F. Macdonald1, B. E. Hayden² and N. I. Zheludev ${ }^{1,3}$ \\ 1. Optoelectronics Research Centre and Centre for Photonic Metamaterials, University of Southampton, Southampton, SO17 1BJ, UK \\ 2. Department of Chemistry, University of Southampton, Southampton, SO17 1BJ, UK \\ 3. Centre for Disruptive Photonic Technologies, School of Physical and Mathematical Sciences \& The Photonics Institute, \\ Nanyang Technological University, Singapore 637371
}

The chalcogenides represent a unique material platform, capable of providing high-index dielectric, plasmonic, 'epsilon-near-zero' (ENZ) or topological insulator properties when the constituent elements are combined in the right proportion. Moreover, they can exhibit reversible, non-volatile structural transitions between solid phases with vastly different electromagnetic properties. We report here on a combinatorial study of the synthesis of plasmonic chalcogenides, including compositions that can be switched between plasmonic and dielectric states.

Plasmonic nanostructures, including photonic metamaterials, are conventionally fabricated from noble metals such as gold and silver, but these suffer from high Ohmic losses, especially in the UV-visible spectral range, which compromise many applications. As singular elemental platforms with optical properties that are readily degraded by impurities, they present no possibility for controlled tuning of electromagnetic response parameters (e.g. via doping). In contrast, chalcogenide semiconductors offer a widely compositionally tuneable alternative materials base in which, to date, only crystalline ternary/quaternary alloys have been seen to present plasmonic properties. Here we show that ultra-high-vacuum deposited thin films of chalcogenide alloys including bismuth telluride (BiTe) and germanium antimony telluride (GeSbTe) exhibit a plasmonic response in their amorphous and/or crystalline states at UV/VIS frequencies, with the real part of relative permittivity $\left(\varepsilon_{1}\right)$ taking negative values over a composition-dependent range of wavelengths extending as low as $250 \mathrm{~nm}$ and as high as $980 \mathrm{~nm}$ [Fig 1a].

Using high-throughput physical vapour deposition and characterization techniques we explore a wide range of alloy compositions (e.g. for the BiTe system, wt\% ratios from 1.6 to 17). Thin film samples are produced, by codeposition of programmed density gradients of the constituent elements, in which composition varies continuously over the substrate. Optical, electronic, structural and thermal properties can then be positionally mapped to composition. Compositional variation is found to provide an effective means of tuning the spectral band over which a material is plasmonic (has a negative value of $\varepsilon_{l}$ ); the corresponding losses $\left(\varepsilon_{2}\right)$; and the wavelengths at which it is an ENZ medium.

The plasmonic character of the chalcogenides is illustrated via the fabrication of subwavelength period 'nanograting' metasurface patterns, which present a highly anisotropic optical response: Under TM-polarized illumination (incident electric field perpendicular to the grating lines) strong nano-grating period-dependent plasmonic absorption resonances in the visible range manifest themselves in the perceived colour of the nanostructured domains [Fig. 1b], while for TE-polarized light the metasurface domains are almost indistinguishable from unstructured BiTe.

In summary, we present the first demonstration of compositionally-tuneable all-chalcogenide material platforms for plasmonic and ENZ metadevices in the UV-visible spectral range. The chalcogenide family includes numerous binary, ternary and quaternary sulphides, selenides and tellurides, offering a uniquely adaptable, CMOScompatible material base for plasmonics with compositionally-controlled, and electrically-/optically-switchable, optical properties.

(a)

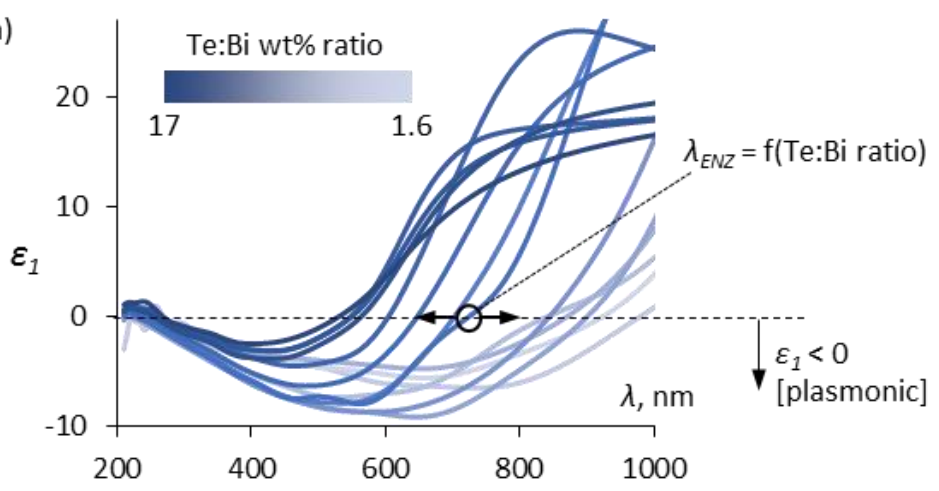

(b)

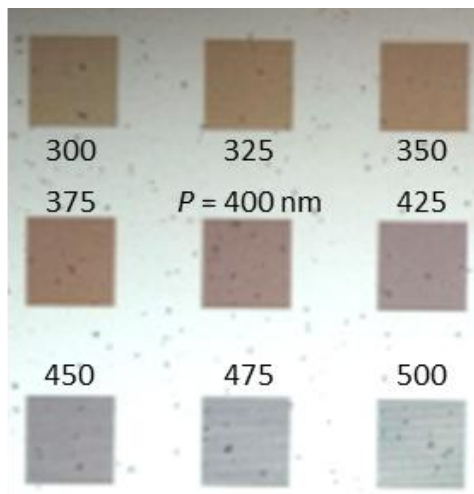

Fig. 1 (a) Real part of the relative permittivity of as-deposited amorphous bismuth telluride [evaluated by variable angle spectroscopic ellipsometry] for a range of elemental $\mathrm{wt} \%$ ratios within a single compositionally graduated thin film sample. (b) TM-polarised optical reflection microscopy image showing plasmonic colours of BiTe nano-grating metasurfaces [periods as labelled; surrounding area is unstructured BiTe]. 